\title{
Evolution of shear fabric in granular fault gouge from stable sliding to stick slip and implications for fault slip mode
}

\author{
M.M. Scuderi ${ }^{1}$, C. Collettini ${ }^{1}$, C. Viti ${ }^{2}$, E. Tinti ${ }^{3}$ and C. Marone ${ }^{4}$ \\ ${ }^{1}$ Department of Earth Sciences, University of Rome La Sapienza, 00185 Rome, Italy \\ ${ }^{2}$ Department of Physical Sciences, Earth and Environment, University of Siena, 53100 Siena, Italy \\ ${ }^{3}$ National Institute of Geophysics and Volcanology (INGV), 00143Rome, Italy \\ ${ }^{4}$ Department of Geoscience, The Pennsylvania State University, University Park, Pennsylvania 16801, USA \\ *Correspondence to: marco.scuderi@uniroma1.it \\ This file includes text, figures and tables divided into seven Data Repository (DR) \\ items:
}

- Table DR1 Summary of experiments and boundary conditions;

- Figure DR1 Evaluation of rate and state friction parameters;

- Figure DR2 Calculation of the dilatancy coefficient

- Figure DR3 Details on fabric evolution from stable sliding to stick-slip;

- Figure DR4 Characterization of the stiffness of the loading system;

- Figure DR5 Growing of frictional instabilities for a typical experiment;

- Figure DR6 The role of applied normal stress in the stiffness of the loading system. 
Table DR1: Summary of experiments and boundary conditions.

\begin{tabular}{|c|c|c|c|c|}
\hline $\begin{array}{c}\text { Experiment } \\
\text { number }\end{array}$ & $\begin{array}{c}\text { Normal stress } \\
\text { (MPa) }\end{array}$ & Spring & Target of the experiment & $\begin{array}{c}\text { Microscopy } \\
\text { analyses }\end{array}$ \\
\hline b266 & 13 & Yes & Stable Sliding & No \\
\hline b267 & 14 & Yes & Stick-slip & No \\
\hline b268 & 13.5 & Yes & Stable sliding & No \\
\hline b371 & 15 & Yes & Stick-slip & SEM \\
\hline b372 & 25 & Yes & Stick-slip & No \\
\hline b390 & 15 & Yes & Stick-slip & SEM \\
\hline b391 & 25 & Yes & Stick-slip & SEM/TEM \\
\hline b416 & 15 & No & Rate \& State friction parameters & No \\
\hline b417 & 25 & Yes & Stick-slip & No \\
\hline b417 & 25 & Yes & Stick-slip & No \\
\hline b418 & 20 & Yes & Stick-slip & No \\
\hline b418 & 20 & Yes & Stick-slip & No \\
\hline b433 & 25 & No & Rate \& State friction parameters & No \\
\hline b511 & 25 & No & Stable sliding $\gamma=4$ & SEM \\
\hline b512 & 25 & No & Stable sliding $\gamma=15$ & SEM \\
\hline b540 & 30 & Yes & Stick-slip & SEM \\
\hline b540 & 30 & Yes & Stick-slip & SEM \\
\hline b541 & 35 & Yes & Stick-slip & SEM \\
\hline b542 & 15 & Yes & Stick-slip & SEM \\
\hline b543 & 35 & Yes & Stick-slip & SEM \\
\hline b544 & 35 & Yes & Stick-slip & SEM/TEM \\
\hline b615 & 35 & Yes & Stiffness during loading/unloading & No \\
\hline b616 & 15 & Yes & Stiffness during loading/unloading & No \\
\hline b617 & 35,15 & Yes & Stiffness during loading/unloading & No \\
\hline
\end{tabular}

All tests were conducted under $100 \%$ relative humidity $(\mathrm{RH})$ to ensure experimental reproducibility. All experiments were run at shear velocity of $10 \mu \mathrm{m} / \mathrm{s}$, except for experiments to retrieve RSF parameter, which included velocity steps tests of 1-3-10 $\mu \mathrm{m} / \mathrm{s}$. 
Figure DR 1: Evaluation of rate and state friction parameters

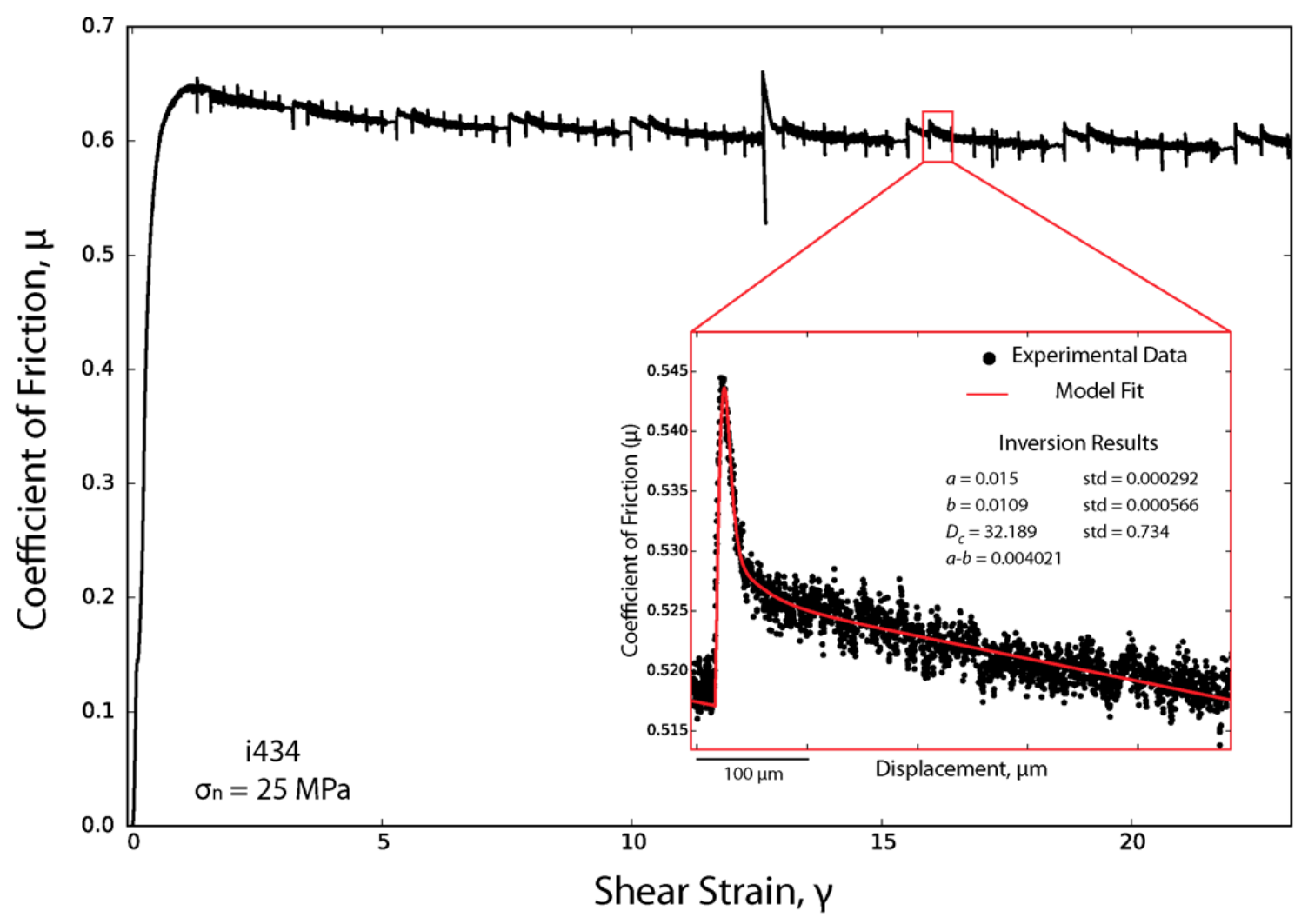

Figure DR1. To characterize the evolution of the rate- and state-friction parameters we performed a series of velocity steps (from 1 to $10 \mu \mathrm{m} / \mathrm{s}$ ) with increasing strain, at normal stresses of 15 and $25 \mathrm{MPa}$. To determine the rate- and state-friction parameters, $(a-b)$ and $D_{c}$, we modelled each velocity step using an iterative singular value decomposition technique, which solves the rate- and state-friction equations using the Ruina evolution law coupled with the elastic interaction of the testing machine (Reinen and Weeks, 1993; Blanpied et al., 1998). The inset in red shows the details of one velocity step with the comparison between experimental data (in black) and the result from the inversion model (red). 
Figure DR2: Calculation of the dilatancy coefficient
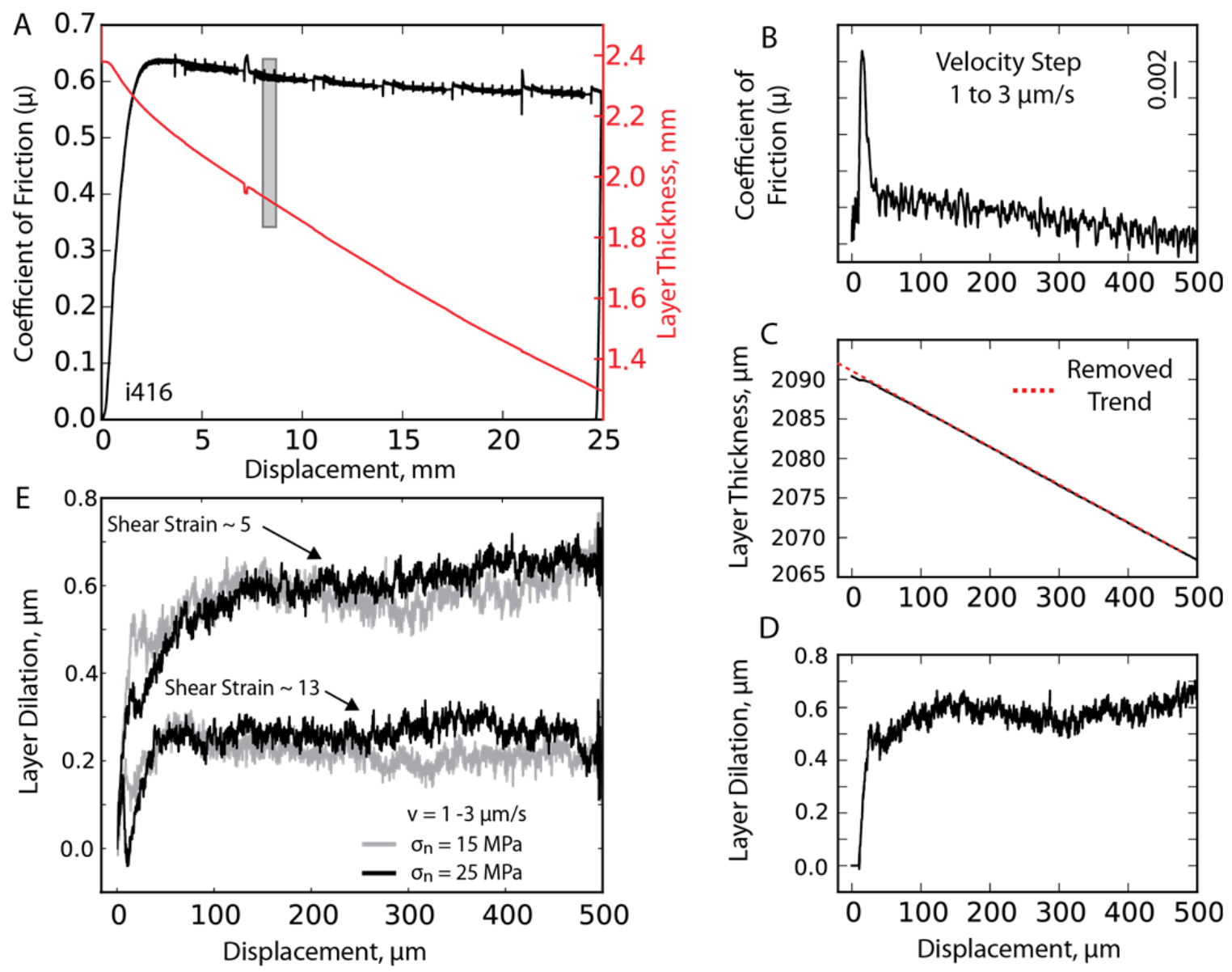

Figure DR2. In order to investigate the degree of shear localization in response to step changes in the imposed slip velocity, we analyzed changes in gouge layer thickness measured at constant normal stress. (Panel A) Data from a typical velocity step test. Note overall geometric layer thinning with superimposed variations. Dilation associated with velocity perturbations was measured after removing geometric layer thinning due to simple shear (Scott et al., 1994). (B) Enlargement of a velocity step (grey box in Panel A), showing the evolution of frictional strength; a linear trend has been removed. (C) Raw data for layer thickness evolution during the velocity step shown in (B). Dashed red line represents the linear trend removed in order to analyze dilation, as in panel D. (D) Detrended data from Panel $C$ showing layer dilation at higher slip velocity. (E) Comparison of layer dilation at two values of shear strain for experiments performed at 15 (grey) and $25 \mathrm{MPa}$ (black) normal stress. Data have been offset so that 0,0 corresponds to the velocity step. Note that for both normal stresses the instantaneous dilation is smaller at higher strain, indicating a greater degree of shear localization 
Figure DR3: Details on fabric evolution from stable sliding to stick-slip

b512, $\sigma_{n}=25 \mathrm{MPa}$ stable sliding at $\mathrm{v}=10 \mu \mathrm{m} / \mathrm{s}$
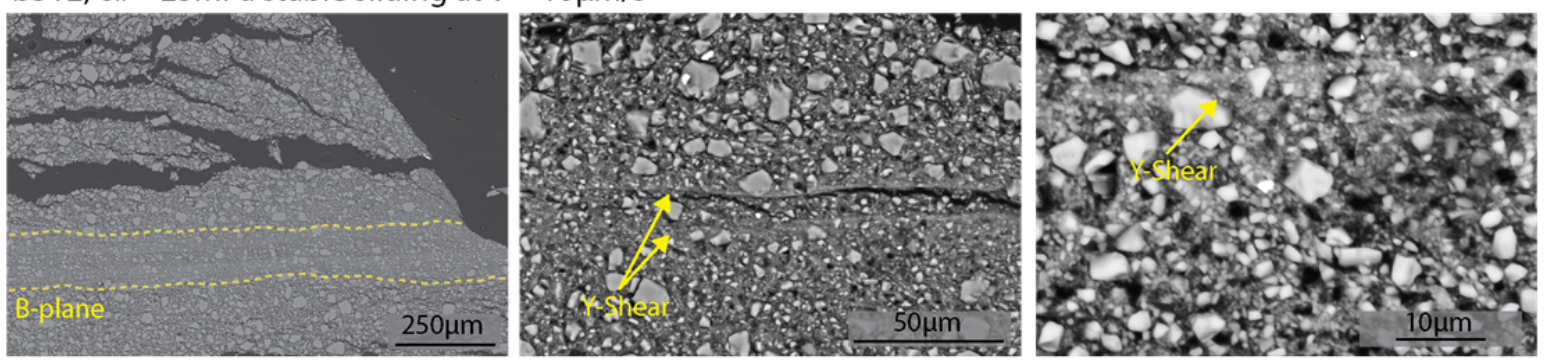

i391, $\sigma_{n}=25 \mathrm{MPa}$ stick-slip average slip velocity $\mathrm{v}=4 \mathrm{~mm} / \mathrm{s}$
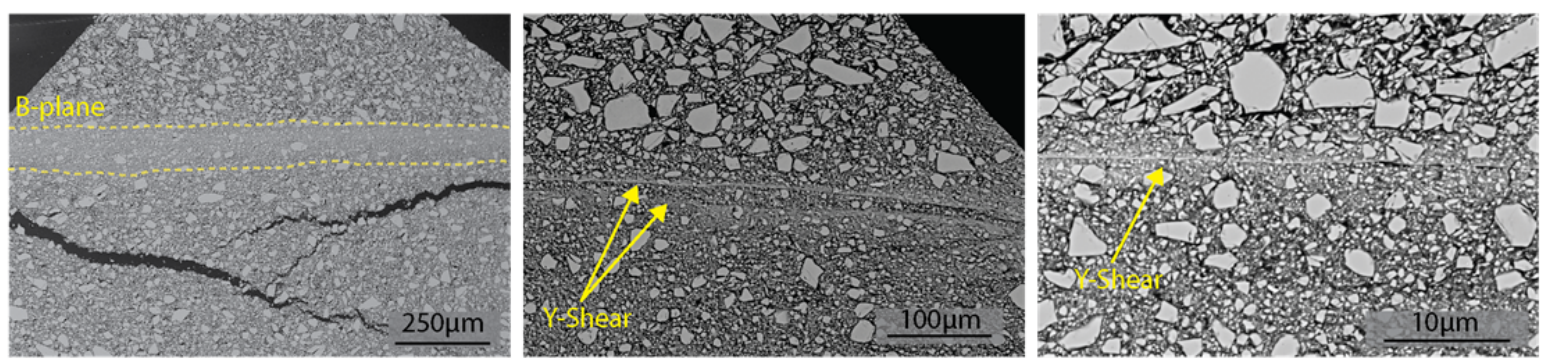

b544, $\sigma_{n}=35 \mathrm{MPa}$ stick-slip average slip velocity $v=3 \mathrm{~cm} / \mathrm{s}$
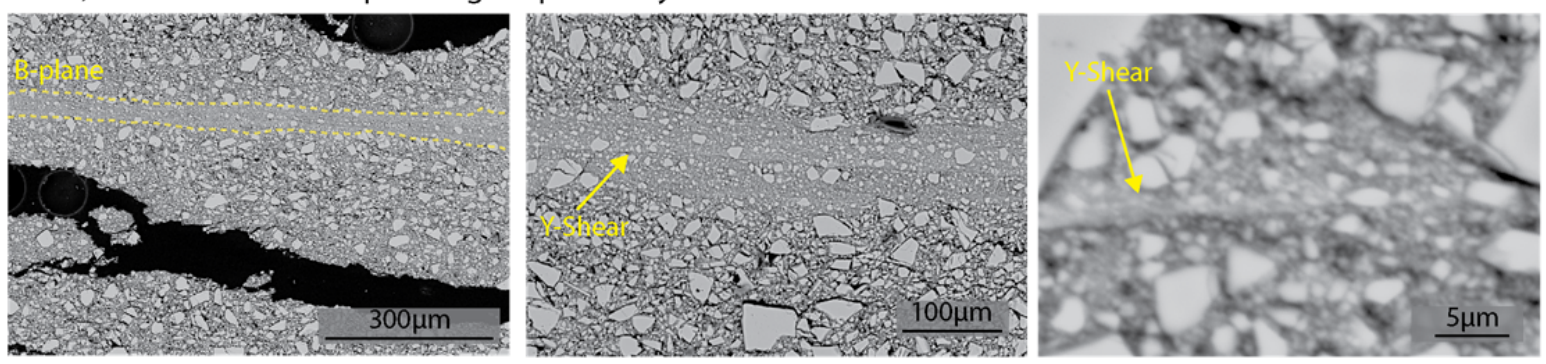

Microstructures for gouge layers sheared at different normal stresses and loading stiffnesses. Lower two rows are layers from dynamic, stick-slip failure. Upper row is a stable sliding experiment. Despite the different stick-slip velocities the microstructures are nearly identical. Note localization along Y-shear planes contained within B shear zones for each case. In these zones of localization the only difference between stable and unstable failure is that for higher stick-slip velocities the B shear zones show a higher level of grain-size reduction. 
Figure DR3 continued
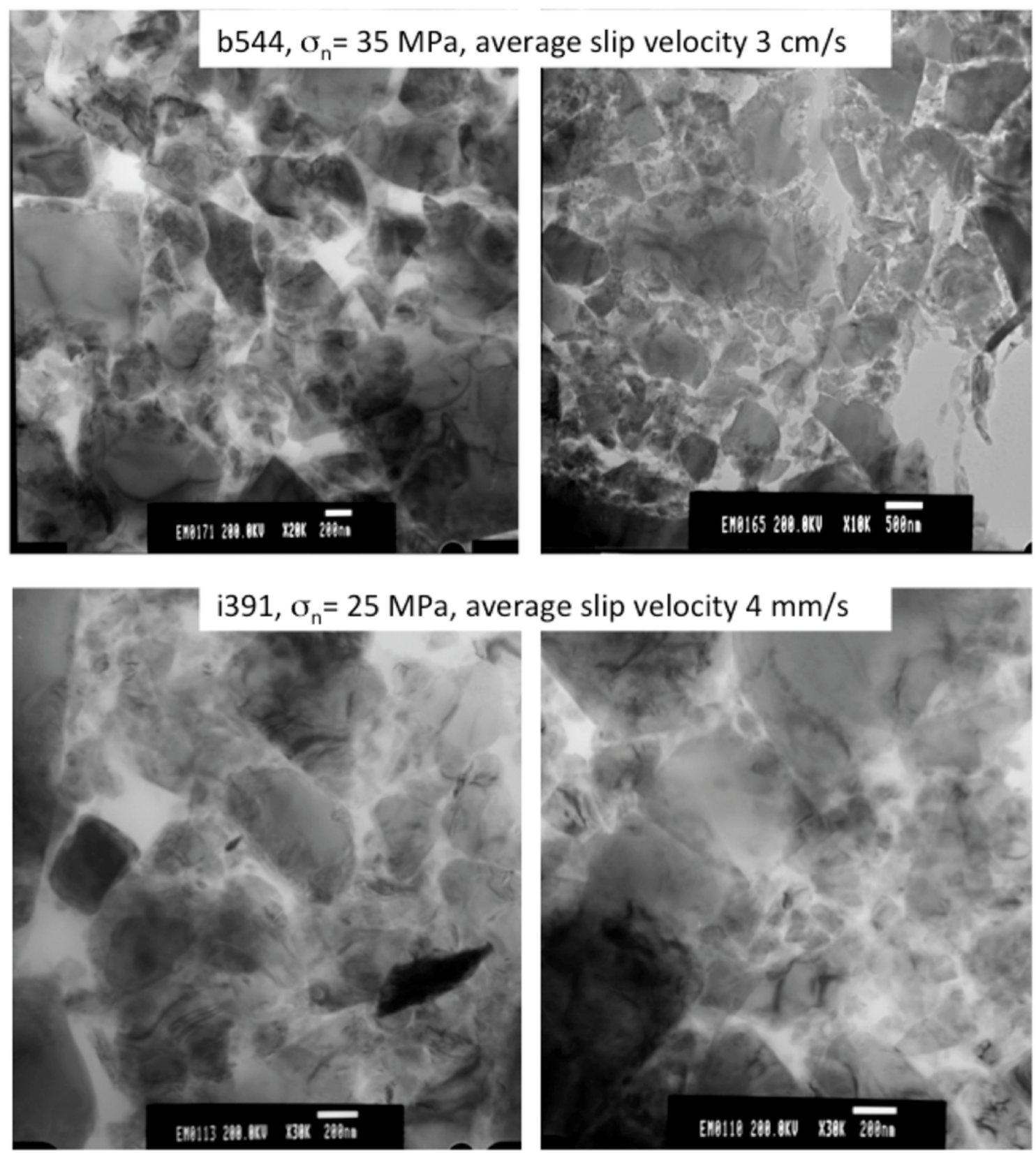

Details of the nanostructure of Y-shear planes for different stick-slip experiments. Nanoscale structures are very similar for the two boundary conditions; each consisting of nanograins, with grain-size of $<500 \mathrm{~nm}$, with intense dislocations. Nanograins likely form by dislocation pile-up during strain accumulation. 
Figure DR3 continued

Starting Material

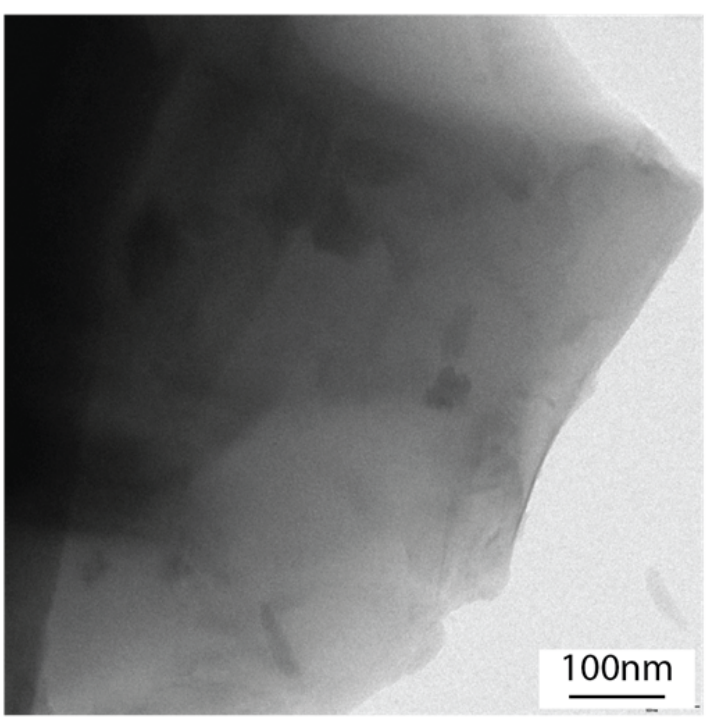

Slipping Zone

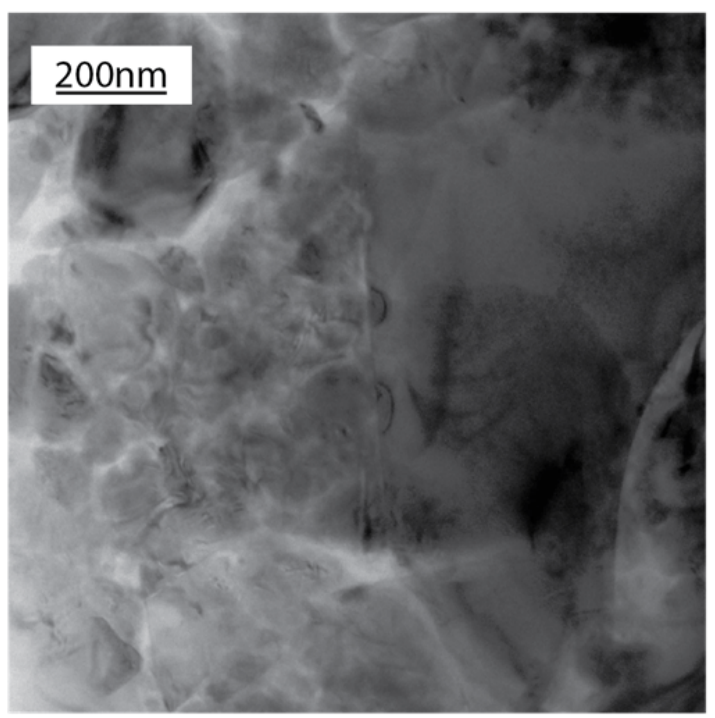

Outside the slipping zone

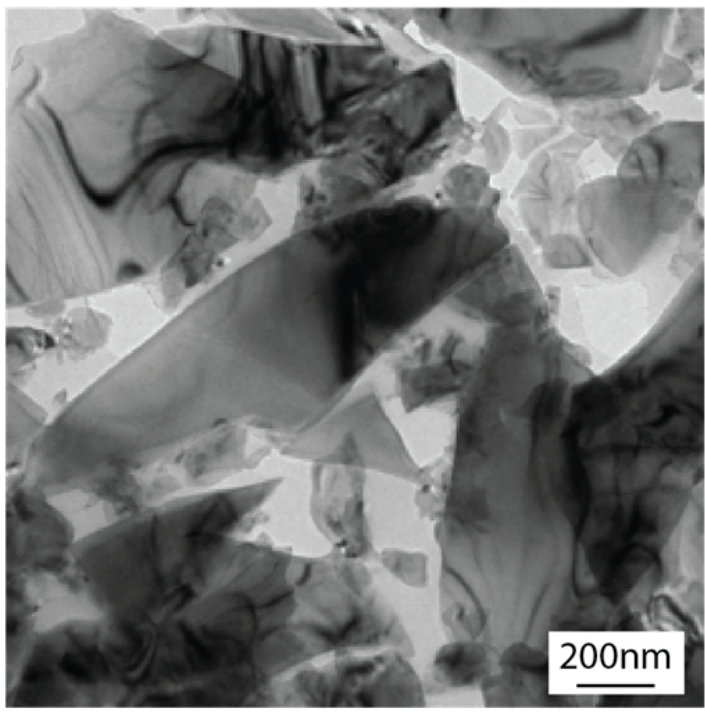

Slipping Zone

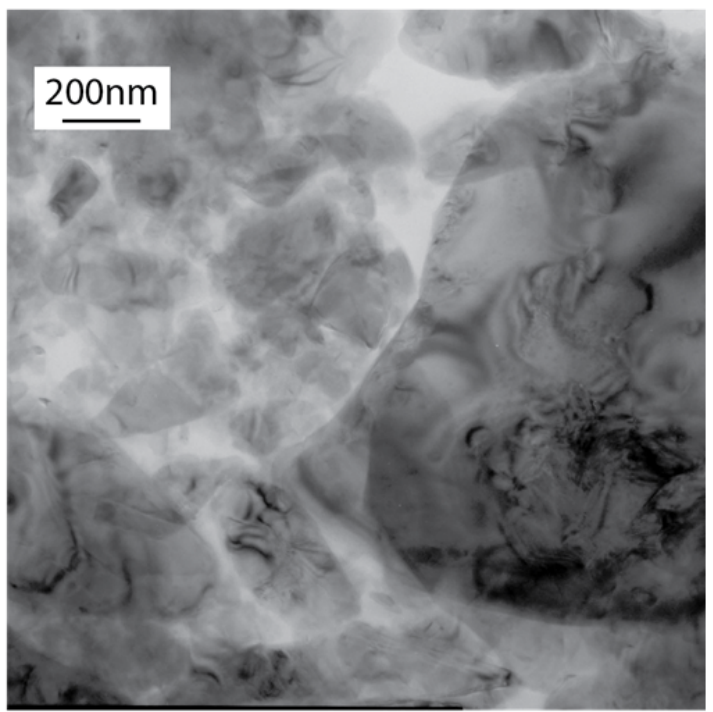

Bright-field phase-contrast images showing the occurrence of dislocations through the socalled dark contrast features. Grains from the starting material (top left) were invariably contrast-free, independently from crystal orientation (i.e., the grains were perfectly homogeneous also during crystal titlting under the TEM beam). Also, grains outside the slipping zone are angular with few dark contrast features indicating low degree of internal deformation. Conversely, grains in the shear zones are more rounded and always characterized by dark contrast features, indicating a high level of internal deformation (i.e. dislocations). 
Figure DR3 continued
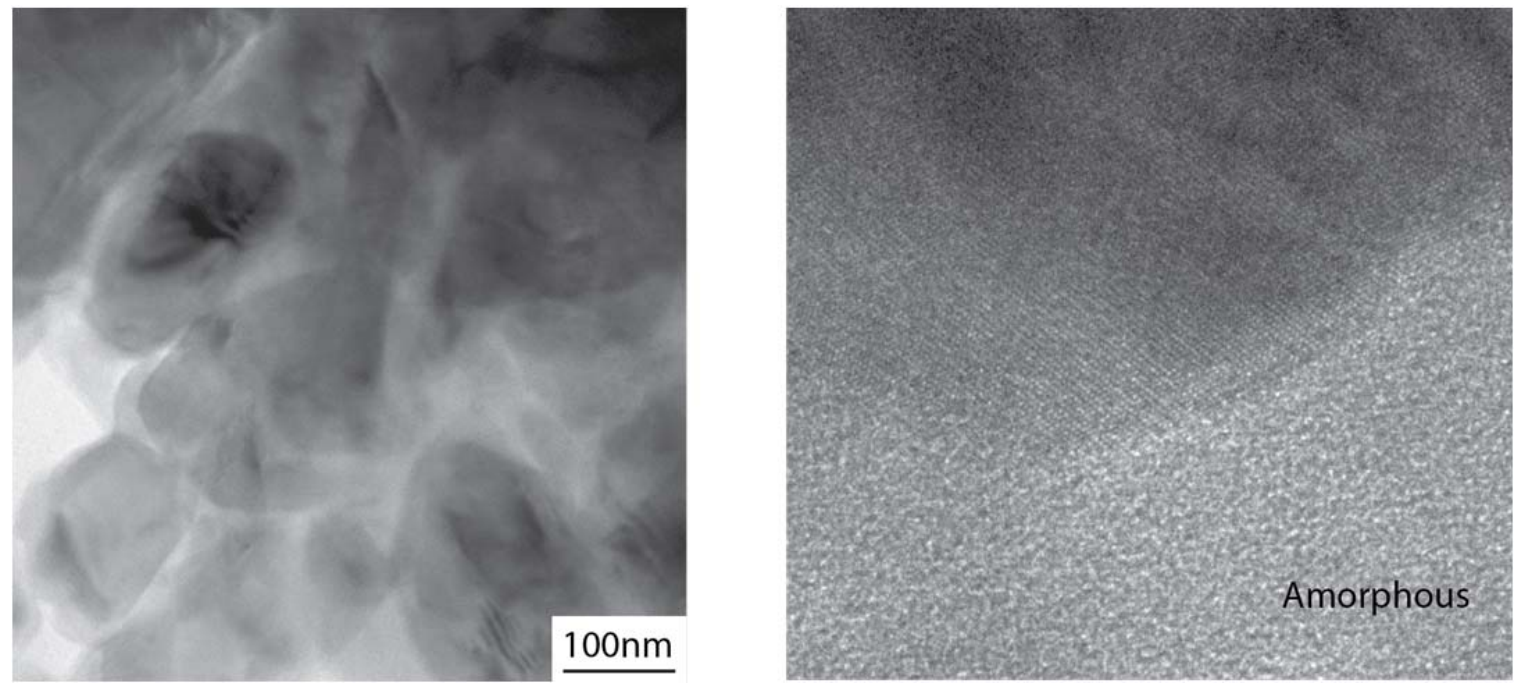

Thin amorphous films surrounding the surface of grains along the highly localized slipping zone. 
Figure DR4: Characterization of the stiffness of the loading system.
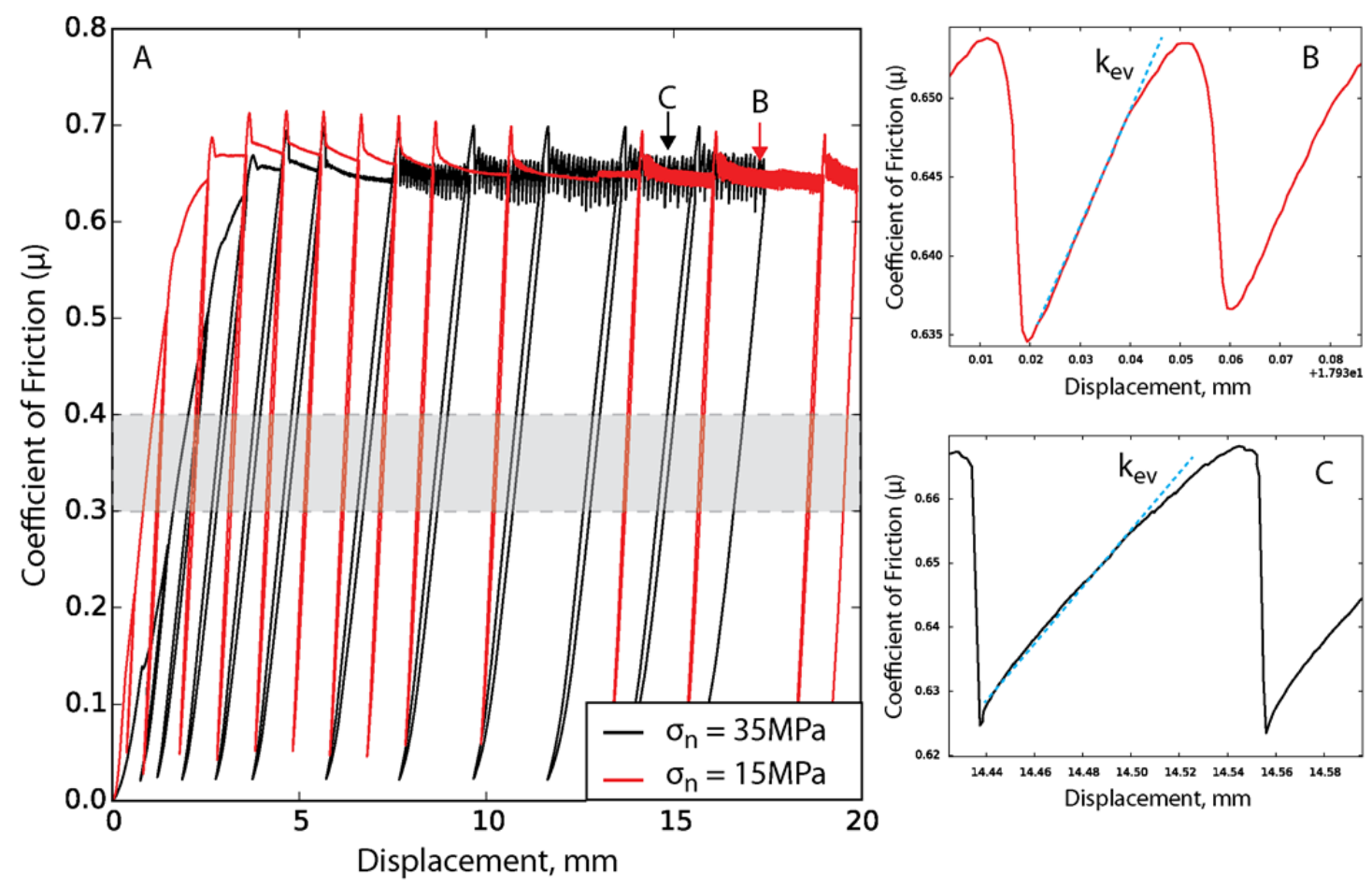

Figure DR4. Data showing the technique used to measure loading stiffness as a function of shear stress and shear strain. The effective loading stiffness is determined by apparatus stiffness, fault gouge stiffness, and normal stress: $k^{\prime}=k / \sigma_{n}^{\prime}$. We evaluate $k$ from load/unload cycles (panel A) and from (panels B and C) the linear, elastic sections of the stick-slip loading curves. Both methods produce similar values, indicating that the shear stress dependence of $\mathrm{k}^{\prime}$ is minor. 
Figure DR5: Growth of frictional instabilities

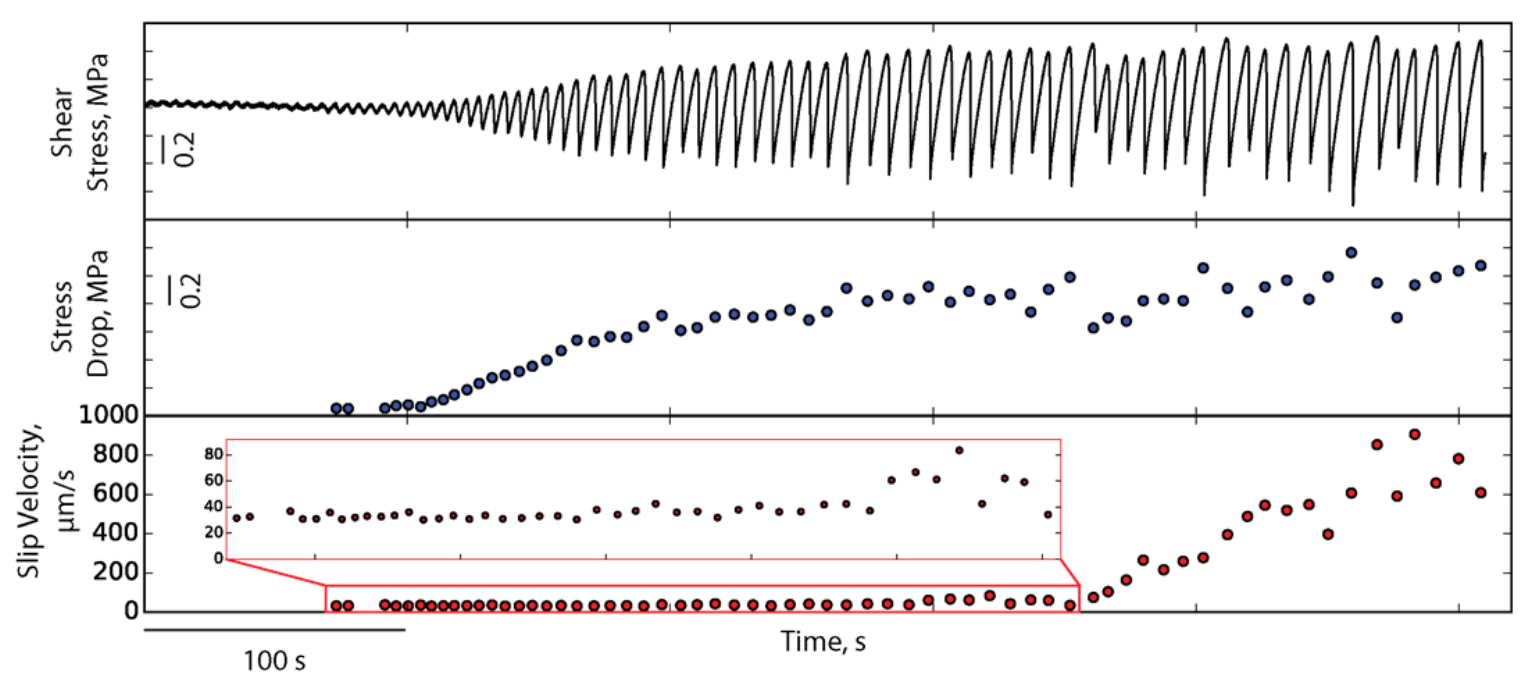

Figure DR5. Here we show an example of the spontaneous emergence of unstable sliding with small stick-slip instabilities that initiate when $K=1$, i.e. near the intersection between black and red curves in figure 3. With increasing displacement we observe a growing phase in stress drop and slip velocity before reaching a nearly steady state, stickslip behaviour. 
Figure DR6: The role of applied normal stress in the stiffness of the loading system.
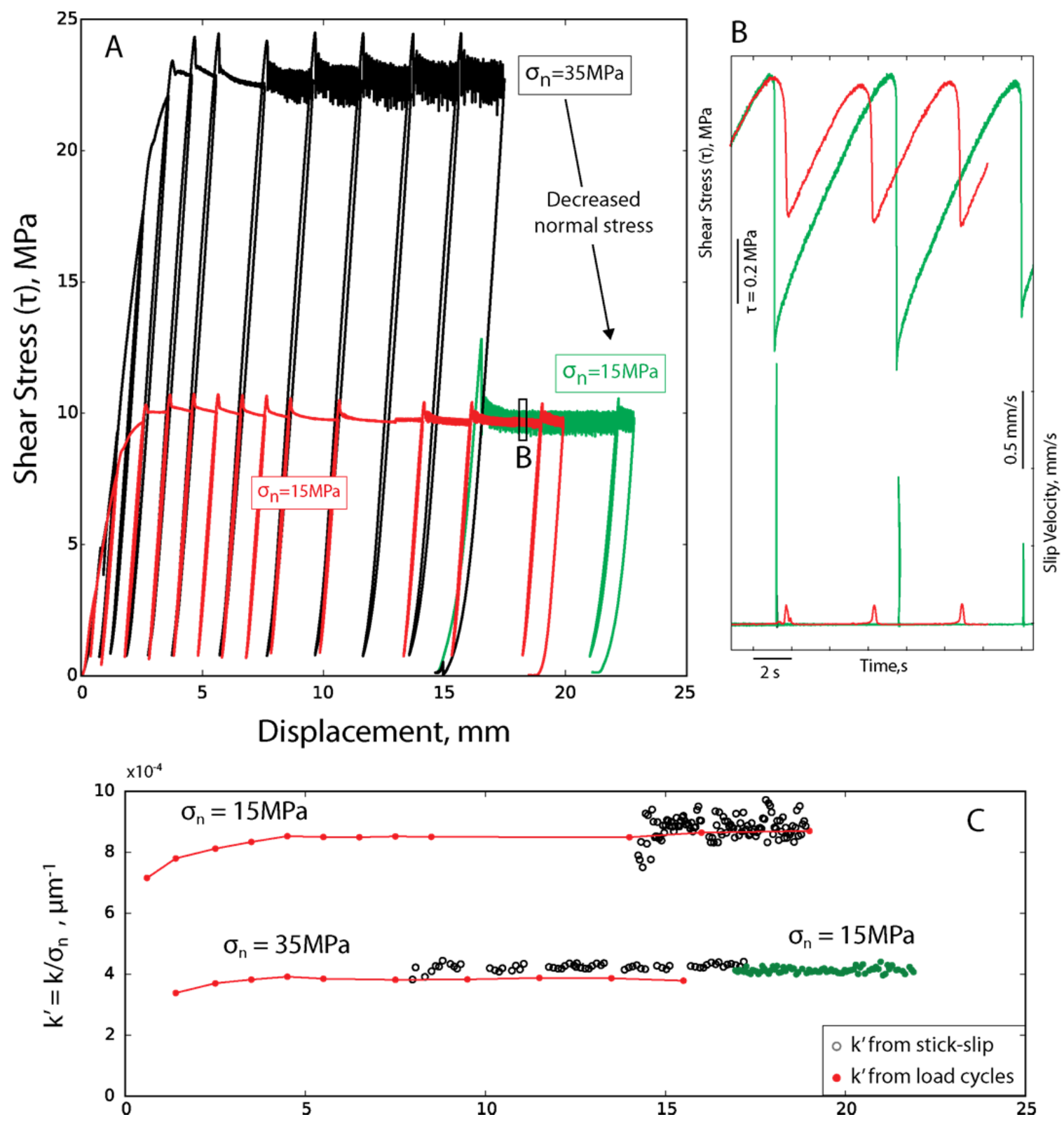

Figure DR6. Elastic stiffness $k$ and its evolution with shear displacement and normal stress. (A) Data for $k$ at two normal stresses as determined from load/unload cycles. Red line shows data for an experiment at 15 MPa normal stress. Black and green lines show an experiment that started at $35 \mathrm{MPa}$ normal stress and was reduced to $15 \mathrm{MPa}$ after $\sim 15$ $\mathrm{mm}$ of shear displacement. Note that sliding is unstable for the run in which normal stress was reduced. These stick-slips are different in comparison to the "standard" stickslips observed at $15 \mathrm{MPa}$ (red curves in figure B) because they have shorter duration of 
the stress drop, larger stress drop (1.0 vs. 0.5 MPa), shorter rise time and faster slip velocity (1.5 vs. $0.15 \mathrm{~mm} / \mathrm{s})$. These faster stick-slip events recorded at $15 \mathrm{MPa}$ result from a decrease of the stiffness of the loading system that it is acquired at $35 \mathrm{MPa}$ and it is maintained when the normal stress is reduced at $15 \mathrm{MPa}$ (Figure C). We interpret this increase in stiffness as a product of fabric development and fault zone stiffness. In particular the lower stiffness of the loading system is likely promoted by a more pronounced grain-size reduction favoured by higher stresses rather than stick-slip velocities, because stable values of stiffness at $35 \mathrm{MPa}$ are reached after $5 \mathrm{~mm}$ of displacement, hence before the onset of stick-slip instabilities

\section{References:}

Blanpied, M.L.; Marone, C.J.; Lockner, D.A.; Byerlee, J.D.; King, D. P. . Quantitative measure of the variation in fault rheology due to fluid-rock interaction. J. Geophys. Res. 103, 9691-9712 (1998).

Reinen, L. \& Weeks, J. Determination of Rock Friction Constitutive Parameters Using an Iterative Least Squares Inversion Method. J. Geophys. Res. 98, (1993).

Samuelson, J., D. Elsworth, and C. Marone (2009), Shear-induced dilatancy of fluid-saturated faults: Experiment and theory, J. Geophys. Res., 114(B12), B12404, doi:10.1029/2008JB006273.

Scott, D., C. Marone, and C. G. Sammis (1994), The apparent friction of granular fault gouge in sheared layers, J. Geophys. Res., 99, 7231-7246. 